Proc. Indian Acad. Sci. (Earth Planet. Sci.), Vol. 95, No. 3, November 1986, pp. 485-503.

(C) Printed in India.

\title{
A review of stochastic dynamic method of weather prediction
}

\author{
P C JOSHI and D R SIKKA \\ Indian Institute of Tropical Meteorology, Pune 411 005, India \\ * Present address: Space Applications Centre, Ahmedabad 380 053, India \\ MS received 26 April 1983; revised 10 February 1986
}

\begin{abstract}
The stochastic dynamic method of weather prediction (SDP) has been suggested recently for better understanding of the numerical weather prediction. The SDP is described using a simple one-dimensional advection equation. The salient features of the method, its scope and limitations, are discussed.
\end{abstract}

Keywords. Stochastic dynamic prediction; weather prediction.

\section{Introduction}

The deterministic method of weather prediction (usually referred as NWP models) requires a knowledge of the initial state of the atmosphere. Time evolution of this state, governed by the dynamical laws, is essentially the aim of these models. In the absence of any contrary evidence the dynamical equations of fluid mechanics are extended to atmospheric systems with apparent modification and parametrization of certain physical features whose explicit inclusion in the dynamical laws is neither possible for want of adequate data nor desirable for want of economy of computational time (e.g. sub-grid scale processes). The dynamical laws are of highly nonlinear nature. Even if we assume that dynamical laws are known perfectly, the knowledge of the initial atmospheric state puts considerable constraints on the weather prediction, because of a significant uncertainty involved in prescribing the atmospheric state uniquely. To start with, the observations of meteorological variables are available at some discrete points which contain some degree of systematic and random errors. Subjective and objective methods of analysis are used to derive the initial state of the atmosphere at regularly spaced grid points from the discrete observations. In the subjective methods, given the same observations, different analysts may arrive at somewhat different values at the grid points. Similarly when different objective methods are utilized to analyze the same data, differences among the grid point values may result due to the interpolation schemes or weighting functions. The differences among the analyses depend to a large extent on the density of the observations available for analyses. Experiments have been reported by several workers for the estimation of the differences among different objective analysis schemes (contours and winds) as applied over mid latitude regions (Kruger 1969, Otto-Bliesner et al 1977, Gustafsson 1981, etc.). Vincent and Borenstein (1980) also conducted experiments concerning variability among subjective analysis schemes over the North American mid-latitudes. Over the Indian tropical region, the objective analysis schemes used follow the successive correction methods (Sikka and Ramanathan 
1970; Mukherjee and Datta 1972; Ramanathan et al 1973). The variability that exists in the tropical wind analyses was discussed by Sikka and Joshi (1983).

Since each analysis (subjective or objective) is consistent with the corresponding set of observed data, there is no a priori method available to say that a particular state is the 'true' initial state of the atmosphere. The non-linear dynamics when applied to prediction problem with two nearly similar initial states may lead to two different states as integration in time advances. It can be seen in some typical nonlinear problems that (Lorenz 1976; 1982) if there is some uncertainty about the initial state, then in finite time this uncertainty may blow upto such a large magnitude that the prediction to satisfactory accuracy becomes impossible. Thus besides the limitation of forecast model, the variability among the so-called imperfect initial state may also impact on the perfectness of the forecast.

The stochastic dynamic method of weather prediction (Epstein 1969a) explicitly considers this inherent limitation. Instead of a single initial state we consider an infinite ensemble of initial states where each of the analyzed state is a member of this ensemble. The time evolution of this ensemble in phase space consistent with dynamical laws then depicts the real representation of the weather prediction. The ensemble is characterized by a probability density function in phase space. The probabilistic initial state so obtained makes the forecast also probabilistic in nature hence the name 'stochastic dynamic' given by Epstein. In this scheme the statistics are used only in describing the uncertainty in the initial state and the evolution of the atmospheric state is governed by deterministic physical laws.

Our objective in this study is to review the stochastical-dynamical weather prediction approach with its possible application to the tropics. Section 2 discusses the basic equations and the methodology of the stochastic-dynamic prediction (SDP). In $\$ 3$ we illustrate the stochastic dynamic prediction method by using a simple example of one dimensional advection equation. Section 4 briefly summarizes the work done by various workers using SDP for both the barotropic and the baroclinic atmospheres. The limitations of the SDP and the possible improvement of these through the Monte Carlo methods are discussed in $\$ \$ 5$ and 6 respectively. The conclusions are summarized in section 7 .

\section{Methodology of stochastic dynamic prediction}

\subsection{Stochastic dynamic equations}

Suppose $N$ parameters $X_{1}, X_{2} \ldots X_{n}$ are needed to describe the atmospheric state. These parameters may be the coefficients of the orthogonal functions in the spectral representation of concerned meteorological field or may be the values of meteorological field at different grid points. Thus the atmospheric state can be represented as a point in the $N$-dimensional phase space. Let us represent this point by the tip of the vector $\mathbf{X}$ in phase space. The ensemble of initial states will consist of a cloud of points in the phase space. The probability density of the ensemble can be represented as $\phi\left(X, t_{0}\right)$. On the basis of the total probability being always one, the following continuity equation could be easily obtained (Gleeson 1968, 1970): 


$$
\frac{\partial \phi}{\partial t}+\sum_{i=1}^{N} \frac{\partial}{\partial X_{i}}\left(X_{i} \phi\right)=0
$$

The solution of (1) is physically not possible for atmospheric system and hence Epstein suggested the following procedure by which one may make use of (1) in prediction problems.

The full probability function contains much more details than of usual interest. Therefore, instead of full function one can study the various moments of this function. The expectation value of a stochastic variable is defined as:

$$
E[f(\mathbf{X})]=\int f(\mathbf{X}) \phi(\mathbf{X}, t) \mathrm{d} \mathbf{X},
$$

where integration is over full phase space. The first and second moments of the distribution are defined as:

$$
\begin{aligned}
& \mu_{i}(t)=\int X_{i} \phi(\mathbf{X}, t) \mathrm{d} \mathbf{X} \\
& \rho_{i j}(t)=\int X_{i} X_{j} \phi(\mathbf{X}, t) \mathrm{d} \mathbf{X} .
\end{aligned}
$$

Instead of the second moment expressed by (4), one is generally more concerned with:

$$
\sigma_{i j}=\rho_{i j}-\mu_{i} \mu_{j}=E\left[\left(X_{i}-\mu_{i}\right)\left(X_{j}-\mu_{j}\right)\right]
$$

which are variances $(i=j)$ and the covariances $(i \neq j)$. Similarly other moments about the mean, viz. $\tau_{i j k}$ (third), $\lambda_{i j k l}$ (fourth) etc. can be defined. Taking the time derivative of (2), making use of (1) one can show that:

$$
\frac{\mathrm{d}}{\mathrm{d} t} E[f(\mathbf{X})]=E\left[\frac{\mathrm{d} f(\mathbf{X})}{\mathrm{d} t}\right] .
$$

Consequently:

$$
\begin{aligned}
\dot{\mu}_{i} & =E\left(\dot{X}_{i}\right) \\
\dot{\rho}_{i j} & =E\left(X_{i} \dot{X}_{j}+\dot{X}_{i} X_{j}\right), \\
\dot{\sigma}_{i j} & =E\left(\dot{X}_{i} X_{j}+X_{i} \dot{X}_{j}\right)-\dot{\mu}_{i} \mu_{j}-\mu_{i} \dot{\mu}_{j} .
\end{aligned}
$$

Also, Lorenz (1963) has shown that every forced dissipative system of which the atmosphere may be presumed to be one, is typified by prognostic equations of the form

$$
X_{i}=\sum_{j, k} a_{i j k} X_{j} X_{k}-\sum_{j} b_{i j} X_{j}+c_{i}
$$

where

$$
\sum_{i, j, k} a_{i j k} X_{i} X_{j} X_{k}=0 \text { and } \sum_{i, j} b_{i j} X_{i} X_{j} \geqslant 0 .
$$

Obviously the forms of $a, b, c$ depend on the dynamics of the atmosphere used. The second term on the right hand side represents the dissipation and the third term 
represents the forcing. Using (10) Epstein derived the equations for the different moments as follows:

$$
\begin{aligned}
\frac{\mathrm{d} \mu_{i}}{\mathrm{~d} t}= & \sum_{j, k} a_{i j k}\left(\mu_{j} \mu_{k}+\sigma_{j k}\right)-\sum_{j} b_{i j} \mu_{j}+c_{i} \\
\frac{\mathrm{d} \sigma_{i j}}{\mathrm{~d} t} & =\sum_{k, l}\left[a_{i k l}\left(\mu_{k} \sigma_{j l}+\mu_{l} \sigma_{j k}+\tau_{j k l}\right)\right. \\
& \left.+a_{j k l}\left(\mu_{k} \sigma_{i l}+\mu_{l} \sigma_{i k}+\tau_{i k l}\right)\right] \\
& -\sum_{k}\left(b_{i k} \sigma_{j k}+b_{j k} \sigma_{i k}\right), \\
\frac{\mathrm{d} \tau_{i j k}}{\mathrm{~d} t} & =\ldots \text { etc. }
\end{aligned}
$$

These equations are the basic stochastic dynamic equations.

\subsection{Moment closure problem}

Equations (12)-(13) reveal that the prognostic equation for each moment contains a moment of next higher order. These equations do not form a closed finite set, which is required of a set of prognostic equations containing nonlinear terms. Even to predict the mean of the distribution, all the moments of the distribution must be known. Accordingly one is forced to make certain assumptions for the higher order moments to close the system. The following assumptions are in use.

\section{2a Third-moment discard: Epstein (1969a) observed the following points}

(i) Expressing $\mu_{i}(t)$ as a Taylor series in time, one finds that the higher order moments enter only in the terms containing higher powers of $\left(t-t_{0}\right)$. Hence the higher order moments may not be important for short range prediction.

(ii) For many operational purposes the first two moments are generally required. Further a knowledge of the first two moments may be a reasonable limit as to what one can expect to derive with confidence, from the observational data.

On the basis of his earlier work, Epstein (1969b) suggested that we can neglect the third moments without sacrificing too much. More specifically he assumed:

$$
\sum_{k, l}\left(a_{j k l} \tau_{i k l}+a_{i k l} \tau_{j k l}\right)=0 .
$$

With this assumption (13) reduces to:

$$
\begin{aligned}
\dot{\sigma}_{i j}= & \sum_{k, l}\left[a_{i k l}\left(\mu_{k} \sigma_{j l}+\mu_{l} \sigma_{j k}\right)\right. \\
& \left.+a_{j k l}\left(\mu_{k} \sigma_{i l}+\mu_{l} \sigma_{i k}\right)\right] \\
& -\sum_{k}\left(b_{i k} \sigma_{j k}+b_{j k} \sigma_{i k}\right)=0
\end{aligned}
$$


Equations (12) and (16) are now prognostic equations in the closed form.

2.2b Quasi-normal-eddy-damped closure: Fleming $(1971 \mathrm{a}, \mathrm{b})$ examined in detail the effects of third moment discard. He did not agree with the assumption that the third moments, which are initially small or zero would not significantly alter the forecast of the first moment until the former becomes large. He suggested that the sum effect of many small third moments may be significant and also the third moments directly affect the second moments. In order to overcome this difficulty Fleming retained the third moments equation and closed the set of equations by making assumptions analogous to the quasi-normal theory used in turbulence studies (Orszag 1970). Assuming initial values are multivariate normally distributed, one finds:

$$
\lambda_{k l p q}=\sigma_{k l} \sigma_{p q}+\sigma_{k p} \sigma_{l q}+\sigma_{k q} \sigma_{l p} .
$$

In a multivariate normal distribution, the third moments are identically zero. However in the approach suggested by Fleming, the third moment equations have been retained and normality has only been assumed for expressing the fourth order moments in terms of the second order moments. Hence the name 'quasi-normal' has been used. However there is always some problem in verifying the validity of the closure schemes. Fleming compared the results of different closure schemes with that obtained by considering a great many samples in a Monte Carlo calculations as used by Epstein (1969a); obviously the Monte Carlo estimates are treated as standard solutions. The quasi-normal closure scheme also showed a significant change from the Monte Carlo solutions after few days. Taking analogy from turbulence theories, Fleming considered the eddy-damping of third moments i.e. the prognostic erquation for third moments is written in the form:

$$
\left.\dot{\tau}_{j k l}=\ldots+\text { [quasinormal }\right]-\kappa \tau_{j k l}
$$

where $\kappa$, the damping coefficient is a positive constant. The damping coefficient would be a function of time, energy density and other statistical quantities; $\kappa$ was taken as an empirical constant by Fleming. This scheme was found to give solutions comparable to those expected from Monte Carlo estimates.

Though the retention of third moments has been necessitated by Fleming's study, most of the studies for the short range prediction until now have been made using the third moment discard, mainly because of avoiding excessive computations.

\subsection{Stochastic analysis of meteorological fields}

The SDP technique has the potential of combining the present and the past information in depicting the 'truer' atmospheric state. The forecasts based on SDP technique for a particular time give the moments of probability density functioncalled 'prior estimates'. These forecasts are obviously made on the basis of past data. The observation at the initial time of the atmospheric state also gives the estimates of first two moments. The forecast estimates can be revised on the basis of observed estimates through the use of Baye's theorem (Epstein 1962). Epstein 
and Pitcher (1972) named this procedure as the stochastic analysis. The steps involved are as follows (Pitcher 1977):

If $\beta$ is a row vector of parameters of dimensions $N$ (implying $N$ terms are retained in the orthogonal expansion), the atmospheric state can be written as

$$
Y=\beta X,
$$

where $X$ is a $P \times N$ matrix of functions (implying observations are made at $P$ points). The SD-prediction precisely gives

$$
\begin{aligned}
& d=E(\beta) \\
& S=\left[(\beta-\mathrm{d})^{\prime}(\beta-\mathrm{d})\right],
\end{aligned}
$$

where a dash represents the transpose of the matrix. These we call as the prior mean and the covariance matrix for $\beta$. The true values and their unbiased estimates are denoted by $\beta$ and $\hat{\beta}$ respectively. The revised judgements, in view of the information contained in observations, called posterior means $\hat{d}$ and covariance $\hat{S}$ can be obtained with the help of Baye's theorem (Epstein 1962). Baye's theorem reads that the posterior probability distribution function is proportional to the product of the likelihood function and the prior probability distribution function. The likelihood function is a conditional probability of the occurrence of the observed data given $\beta$. The likelihood function can always be linked with the probability density of observations errors for which a normal distribution is quite befitting. However, Baye's theorem requires full knowledge of prior probability distribution and only the first two moments of them are known. The normal distribution has the unique property that it can be described by its first two moments. Thus, if only first two moments are considered, the prior distribution, which because of non-information of higher moments, could be taken as the normal distribution. Epstein and Pitcher (1972) argued that with the knowledge of only two moments, we cannot distinguish the 'prior' distribution from a normal distribution. Taking prior also as multivariate normal distribution, it is seen that (for proof see Pitcher 1977):

$$
\begin{aligned}
& \hat{d}=\left(d S^{-1}+Y X^{\prime} \sigma^{-2}\right) \hat{S}, \\
& \hat{S}=\left(S^{-1}+\sigma^{-2} X X^{\prime}\right)^{-1},
\end{aligned}
$$

where

$$
\sigma^{2}=\frac{s^{2}}{P-N}, s^{2}=(y-X \beta)^{\prime}(y-X \beta)
$$

Thus if $P \geqslant N$

$$
\begin{aligned}
& \hat{d}=\hat{S}\left(S^{-1} d+S_{\hat{\beta}}^{-1} \hat{\beta}\right) \\
& S_{\hat{\beta}}=\left[\left(\beta_{0}-\hat{\beta}\right)\left(\beta_{0}-\hat{\beta}\right)^{\prime}\right]
\end{aligned}
$$

i.e. the inverses of the covariance matrices are direct measures of the precision associated with $d$ and $\beta$. In stochastic analyses these matrices become the appropriate weighting factors based on the stochastic weighting factor and the 
particular arrangement of stations in the observing network. If no stochastic forecast is available, then the prior is considered to be vague, or non-informative and $S^{-1}$ is taken as the zero matrix. In this case $\hat{d}$ and $\hat{S}$ reduce to simple least squares solutions.

By making simulated observations of a predefined atmosphere (Epstein and Pitcher 1972) and the real data (Pitcher 1977) it was found that the uncertainty in analyzed values is substantially less than either the uncertainty in the forecast or in the observation. Thus the stochastic analysis offers a unique way of making use of both the past and the present available information for determining the 'true' state of the atmosphere.

\subsection{Procedures of calculations}

The various steps involved in SDP are as follows:

(i) The set of dynamic equation is converted in the generalized canonical form (equation (10)) through some spectral representation.

(ii) Using one of the above mentioned closure approximations, the corresponding stochastic dynamic equations are derived.

(iii) With some suitable numerical schemes the SDP equations are integrated. However for the integration the initial values of the first and the second moments are required. This is one of the difficult problems of SDP. Most of the calculations (Pitcher 1977) are made by using these moments obtained through least squares procedures by fitting the spectral decomposition of equation for the variables to the corresponding observed fields.

The results obtained by various workers are described in the following sections.

\section{A simple illustration of SDP}

From the above discussion it is obvious that in SDP the number of equations is considerably increased in comparison to deterministic prediction. One of the simplest prediction equation in meteorological problems has been the use of one dimensional advection equation (Platzman 1964). Wiin-Nielsen (1979) recently studied the asymptotic behaviour (large time) of the stochastic dynamic solutions of this equation, by taking a Gaussian function for characterizing the ensemble of initial states. Joshi (1981) compared the SD solutions as given by Wiin-Nielsen with the deterministic solutions of the advection equation for illustrating the salient features of SDP. In the following the methodology of SDP is explained using these examples.

The one-dimensional advection equation without the forcing and the dissipation terms is as follows

$$
\frac{\partial u}{\partial t}+u \frac{\partial u}{\partial x}=0
$$

where $u$ is the meteorological variable of interest. For the purpose of SDP, the above equation is transformed into spectral domain as follows: 


$$
u(x, t)=\sum_{n=1}^{\infty} U_{*}(n, t) \sin n K x ; K=2 \pi / L
$$

where an interval $0 \leqslant x \leqslant L$ and the boundary conditions $u=0$ at $x=0$ and $x=L$ have been assumed. Using

$$
\begin{aligned}
& U(n)=U_{*}(n) /\left(2 E_{0}\right)^{1 / 2} \\
& T=\left(2 E_{0}\right)^{1 / 2} K t \\
& 2 E_{0}=\sum_{n=1}^{\infty} U_{*}^{2}(n)
\end{aligned}
$$

and also retaining only two components in the expansion i.e. $X_{u}=U(1)$ and $Y_{u}=U(2)$, the prognostic equation for $X_{u}$ and $Y_{u}$ are found to be

$$
\begin{aligned}
\frac{\mathrm{d} X_{u}}{\mathrm{~d} T} & =\frac{1}{2} X_{u} Y_{u}, \\
\frac{\mathrm{d} Y_{u}}{\mathrm{~d} T} & =-\frac{1}{2} X_{u}^{2} .
\end{aligned}
$$

The variables $X_{u}$ and $Y_{u}$ become the parameters defining the atmospheric state and the above equations as the predictive equations. These equations have analytic solutions in terms of hyperbolic functions. The stability of the deterministic solutions of the equations (29)-(30) is very much sensitive to the initial conditions $\left(X_{u_{0}}, Y_{u_{0}}\right)$. With different initial values of $X_{u_{0}}$ and $Y_{u_{0}}$ and $\sigma_{11}=\sigma_{22}=0 \cdot 1$, $\sigma_{12}=0$ the different phase space trajectories have been derived. The results are shown in figure 1 which also gives the deterministic solutions. The SD solutions correspond to the exact solution of the SD equations.

Figures 1(a) and 1(c) show that the two trajectories are very nearly the same. Thus, as far as the first order moments are concerned, no new information over the deterministic ones has been obtained. In such cases the advantage of the stochastic dynamic prediction lies in the prediction of the second order moments (which has not been shown in the figure) i.e. the uncertainties in the prediction variables are also obtained. However, the stochastic trajectories deviate very much from the deterministic ones (figures $1 \mathrm{~b}$ and $1 \mathrm{~d}$ ). The deterministic trajectories show that both the $X_{u}$ and $Y_{u}$ components are equally significant for most of the time (i.e. the energy is nearly equally distributed in wave number one and two) whereas the stochastic dynamic trajectory clearly shows that for most of the time $Y_{u}$ component (wave number two) is predominant. Since $X_{u}$ and $Y_{u}$ are further used in predicting physical variables, errors in $X_{u}$ and $Y_{u}$ will also lead to errors in the physical variables.

Since the stochastic dynamic solutions have been obtained by considering a small spread (uncertainty) in the initial state, which in actual cases is always present, the SD solutions are expected to be more realistic than the deterministic solutions. Thus from the above simple example, it is evident that in some circumstances the deterministic solutions may give misleading information after some time interval. 


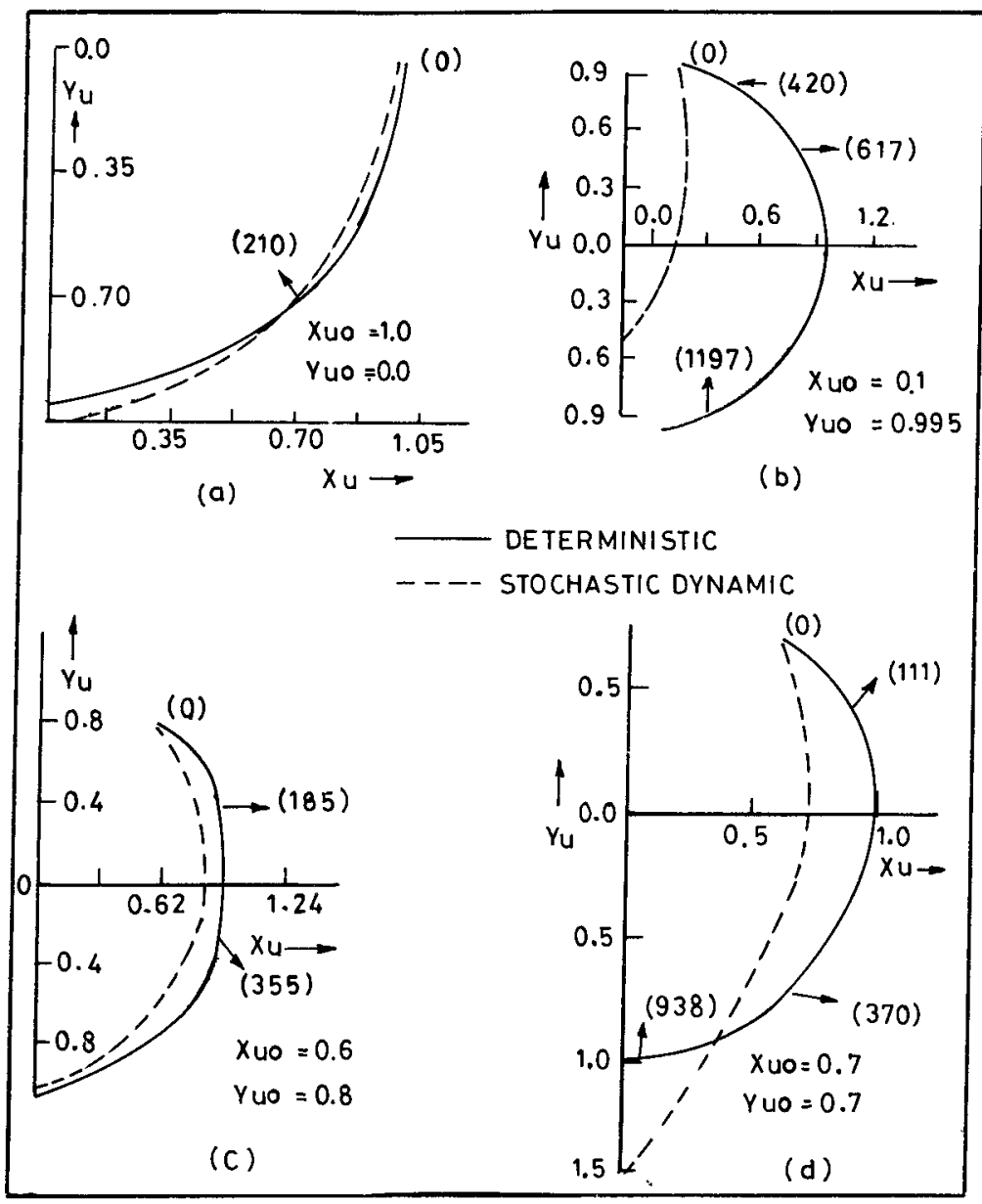

Figure 1. Deterministic and stochastic dynamic trajectories in the phase space for different initial conditions. The entries in the bracket with arrow-mark show the time in hours.

Such situations occur more prominently (Epstein 1969a) for the unstable initial conditions. Interestingly these unstable situations are of greater importance in meteorological phenomena. It should also be noticed that the time interval is crucial. For short times the differences between SDP and deterministic values will be small for a good forecast model and there would be no advantage in using SDP.

\section{Review of other results with the SDP}

\subsection{Barotropic atmosphere}

4.1a Synthetic data The first illustration of SD prediction and their utility was made by Epstein (1969a) by taking the Lorenz's minimum equations (Lorenz 1960) for the prediction and with pre-assigned values of the model parameters. This 
prediction equation is closer to the atmospheric prediction equations with several assumptions and simplifications. However, even after a decade of initiation of SD prediction scheme, most of the studies in SD prediction use these minimum equations, mainly because they are simplest in nature and their deterministic solutions are available in the analytical forms.

Assuming a simplified barotropic atmosphere and a spatial domain of dimension $(2 \pi / k, 2 \pi / l)$ over which the double Fourier expansion of vorticity field has been made viz.

$$
\nabla^{2} \psi=A_{1} \cos l y+A_{2} \cos k x+2 A_{3} \sin l y \sin k x
$$

Lorenz obtained the following prognostic equations for the parameters

$$
\frac{\mathrm{d} A_{1}}{\mathrm{~d} t}=c_{1} A_{2} A_{3} ; \frac{\mathrm{d} A_{2}}{\mathrm{~d} t}=c_{2} A_{1} A_{3} ; \frac{\mathrm{d} A_{3}}{\mathrm{~d} t}=c_{3} A_{1} A_{2}
$$

The first term in (31) represents the zonal flow and the second and the third terms represent the eddies superimposed over this. These equations are called Lorenz's minimum equations. Solutions of these equations are elliptic functions. Epstein (1969a) made the stochastic dynamnic calculations (with third moment discard) for these equations with two extreme situations - one in which initial conditions are stable and the other in which they are unstable. The stable and unstable conditions are determined by relative magnitudes of different coefficients i.e. $A_{1}, A_{2}$ and $A_{3}$. For a stable condition $A_{1}=0.12, A_{2}=0.24, A_{3}=0.0$ as initial condition with $\sigma_{i i}=10^{-4}(3 \mathrm{hr})^{-1}$ and $\left.\sigma_{i j}=0 \cdot 0 i \neq j\right)$, the results are shown in figure 2 . Monte Carlo solution with a sample size of $N=500$ is treated as standard to compare the SD solutions. On the scale of the graph it is not possible to distinguish between the SD solutions and Monte Carlo solutions. The deterministic solution is also very close to these solutions. In this example, the initial uncertainties were relatively small, i.e. they did not have severe effects on the forecasts of the parameters. In

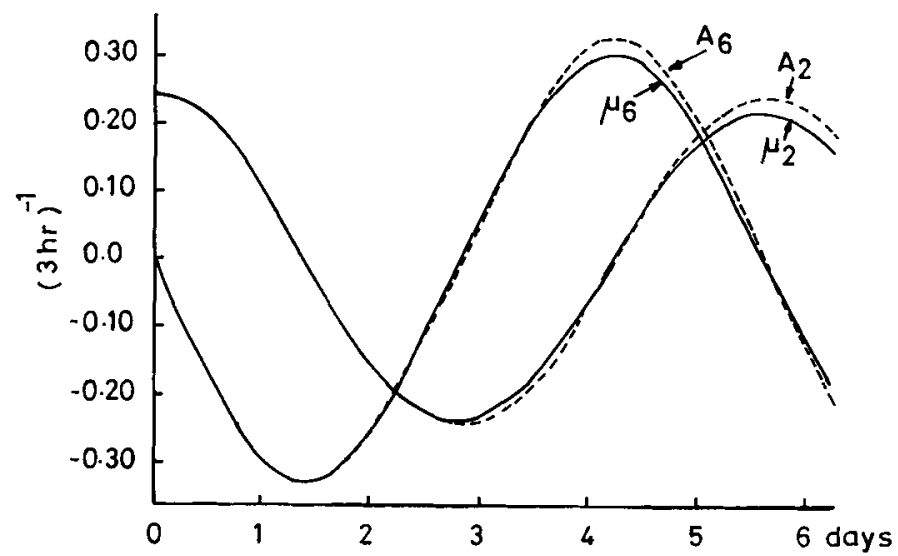

Figure 2. SDP solutions for $\mu_{2}$ and $\mu_{3}$ and the deterministic solutions for $A_{2}$ and $A_{3}$ for stable initial conditions (after Epstein 1969a). 
such cases the utility of the stochastic procedure lies in the information about the uncetainties.

For an unstable initial condition $A_{1}=0.12, A_{2}=0.00, A_{6}=0.66$ and all covariances as zero and variances as earlier, the representative result for the parameter $A_{1}$ is shown in figure 3 . The sign of $A_{1}$ becomes negative after three days. The SDP solution $\mu_{1}$ remains positive throughout the period. The differences between the two stochastic solutions (i.e. SD and Monte Carlo) are certainly real after the third day. The difference may be attributed to the third moment discard. The deterministic solution shows departures from the Monte Carlo solution after $24 \mathrm{hr}$ and is very misleading after about 3 days. Epstein also showed that the solutions for $A_{2}\left(\mu_{2}\right)$ and $A_{3}\left(\mu_{3}\right)$ tend to emphasize these differences. It should be noted here that the implication here is not this that the forecast is wrong, only that it is a poor forecast.

Assuming that for sufficiently small times the deviations from the deterministic results are small, Laurman (1978) linearized the SDP equations using small perturbation approximations. This appproximation is applied to both the cases of Epstein (i.e. applying to Lorenz's minimum equations) and it is seen that a substantial reduction in computational time is possible. However, as described earlier, for smaller times SDP hardly gives any new information over deterministic formulation.

\section{1b Real data}

An attempt for the real atmospheric data was first made by Pitcher (1977). For predicting the height of $500 \mathrm{mb}$ level he used a simple barotropic vorticity equation

$$
\frac{\partial}{\partial t}\left(\zeta+\frac{r^{2}}{a_{0}^{2}} \psi\right)=\mathbf{V} \cdot \nabla(\zeta+f+c h)
$$

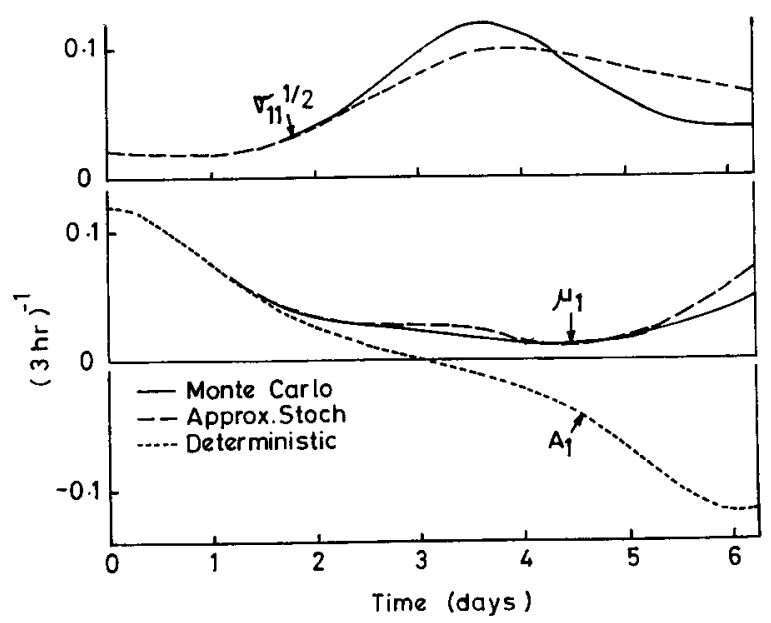

Figure 3. SDP solutions for $\mu_{i}$ and $\sigma_{i i}^{1 / 2}$ and the deterministic solution for $A_{1}$ for unstable initial conditions (after Epstein 1969a). 


$$
\text { where } \begin{aligned}
\psi & =\text { stream function } \\
\zeta & =\text { vertical component of relative vorticity } \\
f & =\text { coriolis parameter } \\
\mathbf{V} & =\text { horizontal wind } \\
h & =\text { the smoothed terrain height } \\
c & =\text { parameter measuring the effectiveness of the } \\
& =\text { orographically induced vertical velocity } \\
r & =\text { parameter to reduce retrogression of long waves } \\
a_{0} & =\text { earth's radius }
\end{aligned}
$$

The vorticity equation is transformed into spectral form by expanding $\psi$ and $h$ in terms of spherical harmonics

$$
\psi(\phi, \theta, t)=\sum_{m=-J}^{J} \sum_{n=|m|}^{|m|+J} a_{0}^{2} \psi_{n}^{m}(t) Y_{n}^{m}(\phi, \theta)
$$

and similarly for $h(\phi, \theta)$, where $\psi_{n}^{m}$ is an expansion coefficient, $\theta$ the longitude, $\phi$ the latitude, $Y_{n}^{m}$ are spherical harmonics, $a$ is the radius of the earth and $J$ is the wave number at which expansion has been truncated. Restricting the studies to the northern hemisphere, Pitcher has taken the period of 1-4 December 1969. Geopotential heights were available from approximately 450 radiosonde stations. Less than $10 \%$ of the data was located south of $20^{\circ} \mathrm{N}$. The method of least squares was used to extract the moments directly from observations. In order to stabilize the least squares fit in the tropical region, it was found necessary to insert artificial data there. Approximately twenty points were added and assigned climatological values appropriate for the month of December. The expansion was terminated at $J=10$. This limited the spectral coefficients to 105 . Thus a total of 5670 numbers, the parameters and their variance-covariance information, formed the initial conditions for a stochastic prediction. The height fields were presented as the least squares estimated, the stochastically forecasted and the stochastically analyzed (as described in \$2.3) fields for all the four days. It was found that the uncertainty in the stochastically analyzed height fields was much less than the observed height field. The standard error of the forecast (second moment) was greater than the corresponding uncertainty associated with the observed expected state. Qualitatively, this would indicate that the forecast expected state would be given less weight than the least-squares estimations in arriving at a stochastically analyzed expected state. They also found that at some points the uncertainty in the forecasted field was less than the uncertainty in the initial field. This result is possible and is a manifestation of the detailed influence of the correlations between the spectral amplitudes in the model. The uncertainty of each model parameter is not necessarily a monotonically increasing function of time throughout the forecast period. The examination of the standard error field and the observed data field after few days also revealed that some times the subsequent analysis placed more credence in the forecast expected state. They concluded that the validity of the analyzed uncertain information depends crucially upon the extent to which the uncertainty in the observed and forecast expected states is faithfully represented. 


\subsection{Baroclinic Atmosphere}

Fleming (1971a,b) made the SD calculations using a baroclinic model by considering a simple two-layer model having 28 degrees of freedom.

The two-level model used was taken from Lorenz (1965). In this model the basic equations are the quasi-geostrophic vorticity equation and the thermal equation that could be written with $x, y$ as horizontal coordinates and pressure $p$ as the vertical coordinate. The equations of the model in spectral form are:

$$
\begin{aligned}
\dot{\psi}_{i}= & \sum_{\substack{j, k=1 \\
k>j}}^{\infty}\left[a_{i}^{-2}\left(a_{j}^{2}-a_{k}^{2}\right) c_{i j k}\left(\psi_{j} \psi_{k}+\theta_{j} \theta_{k}\right)\right]-\kappa^{\prime}\left(\psi_{i}-\theta_{i}\right) \\
\dot{\theta}_{i}= & \sum_{\substack{k, k=1 \\
k>j}}^{\infty}\left[c_{i j k} \frac{\sigma_{0}\left(a_{j}^{2}-a_{k}^{2}\right)+1}{\sigma_{0} a_{i}^{2}+1} \theta_{j} \psi_{k}\right. \\
& \left.+c_{i j k} \frac{\sigma_{0}\left(a_{j}^{2}-a_{k}^{2}\right)-1}{\sigma_{0} a_{i}^{2}+1} \psi_{j} \theta_{k}\right]+\frac{\kappa^{\prime} \sigma_{0} a_{i}^{2}}{\sigma_{0} a_{i}^{2}+1} \psi_{i} \\
& -\frac{\sigma_{i} a_{i}^{2}\left(\kappa^{\prime}+2 \kappa^{\prime \prime}\right)+h}{\sigma_{0} a_{i}^{2}+1} \theta_{i}+\frac{h}{\sigma_{0} a_{i}^{2}+1} \theta_{i}^{*}
\end{aligned}
$$

where $\psi$ and $\theta$ are the non-dimensional dependent variables representing the mean wind and mean potential temperature and $C_{i j k}$ are constants depending upon the choice of the orthogonal functions used to represent the space domain. Qualities had been made dimensionless by appropriate scale factors including a length $L$ and a time $f^{-1}$ ( $f$ is the coriolis parameter assumed constant here). A frictional drag with coefficient $2 \kappa^{\prime}$ is introduced at the lower surface, proportional to the velocity in the lower layer. A frictional drag at the boundary separating the two layers with coefficient $\kappa^{\prime \prime}$ is introduced proportional to the difference between the temperature of the lower layer and a preassigned temperature field $\theta^{*}$, characterized by a coefficient $2 h$. A coefficient for heat exchange between the layers cancels out when $\sigma$ (a measure of static stability) is considered constant. The choice of the functions depends upon the geometry of the space domain in which the model equations are applied. In the present case the functions were given over an infinite channel of width $\pi L$ having walls at the surfaces $y=0$ and $y=\pi L$ where the flow in the channel is periodic along the length with a specified fundamental wavelength. The model had 28 variables (14 orthogonal functions for $\psi$ and $\theta$ ).

Fleming studied the stochastic dynamic effects by considering the energetics of the atmosphere. For the deterministic model referred above the kinetic energy $K$ and the available potential energy (hereafter referred to as APE) $A$ could be derived as:

$$
K=\frac{s}{2} \sum_{i} a_{i}^{2}\left(\psi_{i}^{2}+\theta_{i}^{2}\right)
$$




$$
A=\frac{s}{2 \sigma} \Sigma \theta_{i}^{2}
$$

However if we apply the stochastic dynamic equations to the above model, the energies are given by

$$
\begin{aligned}
& K=\sum_{i} \frac{a_{i}^{2}}{2}\left[\psi_{i}^{2}+\theta_{i}^{2}+\text { variance }\left(\psi_{i}\right)+\text { variance }\left(\theta_{i}\right)\right] \\
& A=\sum_{i} \frac{1}{2_{\sigma}}\left[\theta_{i}^{2}+\operatorname{variance}\left(\theta_{i}\right)\right]
\end{aligned}
$$

Thus it appears that the stochastic dynamic equations provide for a partitioning of the energy. There is a 'certain' energy associated with the expected components of the ensemble and 'uncertain' energy associated with the variances. The initial uncertain energy may represent, in part, some of the actual energy of the atmosphere; that is, energy is lost by smoothing the data or turbulent energy, or any small-scale energy unresolved by the deterministic initial conditions. Thus the quantity $\sum_{i} \frac{1}{2} a_{i}^{2}\left(\psi_{i}^{2}+\theta_{i}^{2}\right)^{\cdot}$ is the certain kinetic energy; $\sum_{i} \frac{1}{2} a_{i}^{2}\left[\operatorname{variance}\left(\psi_{i}\right)+\right.$ variance $\left.\left(\theta_{i}\right)\right]$ is the uncertain kinetic energy, $\Sigma \theta_{i}^{2} / 2 \sigma$ is the certain APE; and $\sum$ Variance $(\theta) / 2 \sigma$ is the uncertain APE. It is to be mentioned here that $\psi_{i}$ and $\theta_{i}$ in (37) and (38) are deterministic stream function and potential temperature whereas they refer as ensemble mean values in (39) and (40).

Fleming derived the expressions for different energy parameters viz. the zonal APE, the eddy APE, the zonal K.E. and eddy K.E. with both 'certain' and uncertain components as well as generation, dissipation of different kinds of energies and also the various conversions from one kind of energy to the other. His results showed that the third moments do not affect energy conservations but they affect energy conversions between uncertain components. The change in 'uncertain components' eventually lead to altering the forecast of the mean. Considering various stable and unstable initial conditions and their certain and uncertain components of energies, Fleming found that the third moment discard stochastic dynamic forecast of the mean of the stream function and potential temperatures are different from more accurate Monte Carlo calculations. As an example the stream function coefficient corresponding to wave number 4 and its prediction is shown in figure 4. Here the SDP corresponds to third moment discard approximation. The deviations between Monte Carlo and the SDP for this component start becoming important after 13 days. Figure 5 shows the results for the same component using eddy-damped quasinormal scheme described in $\$ 2.2$. The calculations of SDP now seem to be in very close agreement with the Monte Carlo upto 18 days. In a subsequent study Fleming (1973) investigated the balance of wind and the mass field in the phase space by suggesting a stochastic balance equation. He found that the adjustment of the fields could not take place with the third moment discard approximation. 


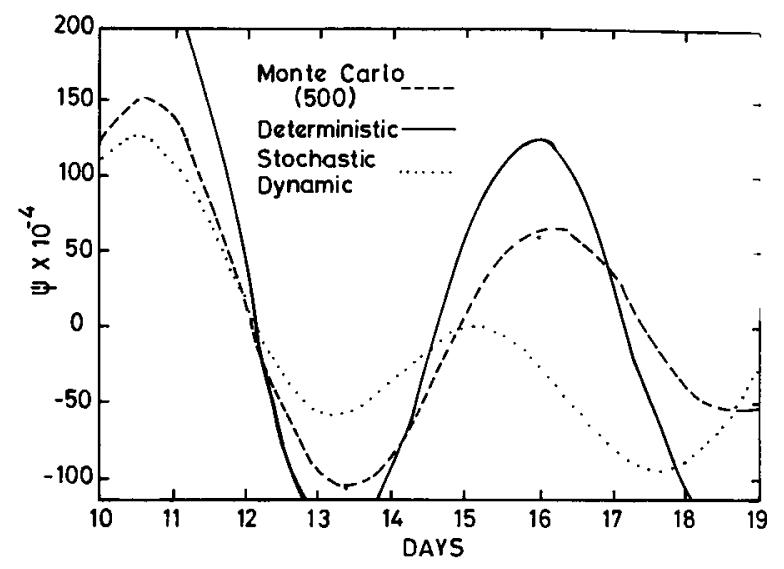

Figure 4. Comparison of SDP (third moment discard) with the Monte Carlo calculations in a baroclinic atmosphere for a stream function component corresponding to wave number 4 (after Fleming 1971a)

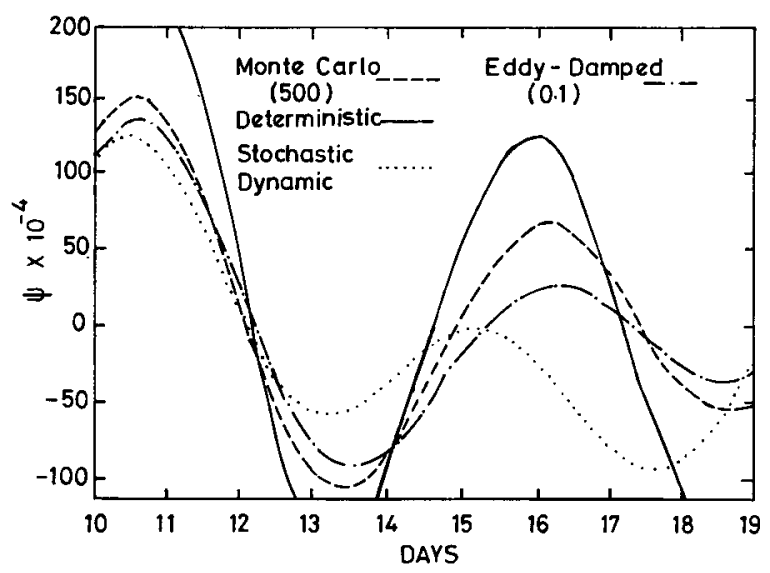

Figure 5. Same as in figure 4 but with eddy damped quasi-normal closure scheme in SDP (after Fleming 1971a)

\section{Limitations of SD predictions}

Except for the study of low order systems, the approach outlined above has the following rather severe limitations if application to current NWP models is contemplated.

(i) Foremost among these is the excessive computation time required over a conventional forecast for a system with reasonably large number of parameters. In an $N$-parameter system the total number of equations (corresponding to the first and second moments) comes out to be $N(N+3 / 2)$ i.e., an increase in computation time of roughly $N$ orders of magnitude. 
(ii) The above formulation is limited to quadratically nonlinear systems and while this appears to be the dominant nonlinearity in atmospheric prediction models, a reformulation taking into account higher order nonlinearities, as are present in certain local physical processes would pose some severe mathematical difficulties and an extra computational burden.

(iii) SDP models ought to be spectral rather than grid point to reduce the total number of covariance quantities.

(iv) The SDP equations require some closure approximation. Various closure approximations made to solve the system may not be valid for some situations.

\section{Monte Carlo forecasting}

Many of the practical difficulties associated with the solution of the SD equations may be substantially alleviated, if not eliminated entirely, by utilising a Monte Carlo approach. Leith $(1974,1980)$ advocated such an alternative and investigated the theoretical skill of the Monte Carlo procedure by application to a twodimensional turbulence model. Instead of predicting the moments of the forecast ensemble directly, one draws a sample of $m$ members from the initial ensemble and integrates each member forward in time with the governing dynamical equation. Later, the $m$ members continue to serve as a sample drawn from the evolved ensemble and the ensemble moments may be estimated from averages over the sample still consisting of original $m$ members. Of course such a calculation could be repeated with a different sample, thereby obtaining a different set of statistical estimates. The hope, however, is that we may choose, within the limits of computer resources, a sample size $m$ sufficiently large to produce stable statistics. Thus we have

$$
\left.\mu_{i}=\bar{X}_{i}, \sigma_{i j}=\frac{m}{m-1} \overline{\left(X_{i}-\mu_{i}\right)\left(X_{j}-\mu_{j}\right.}\right)
$$

where overbar denotes an average over the sample of $m$ members.

The total computation involved is proportional to $m N$, but if $m \ll N$ we may capture the advantage of SDP without the penalty of excessive arithmetic.

Other limitations of the stochastic-dynamic system of equations discussed above are not present with the Monte Carlo procedure. However, in the procedure advocated the problem of moment closure is replaced by the sampling problem in deciding what value to choose for $m$, which is done essentially by experimentation.

Pitcher (1979) investigated the design of the Monte Carlo forecasting technique by taking a case study of generating an initial sample. He considered a relatively straightforward manner of generating an initial sample that possess a statistical structure reflecting inhomogeneity and spatial correlations among analysis errors. Assuming existence of an unbiased initial state vector $\mathbf{X}$, the initial sample was generated by choosing $\mathbf{X}_{i}=\mathbf{X}+\boldsymbol{\varepsilon}_{i}(i=1,2, \ldots m)$; where $\boldsymbol{\varepsilon}_{i}$ were chosen at random. Choosing spherical harmonics as basis functions the error field $\varepsilon_{i}$ were represented as 


$$
\begin{aligned}
\varepsilon & =\sum_{m, n}\left(A_{n}^{m} \cos m \lambda+B_{n}^{m} \sin m \lambda\right) P_{n}^{m}(\mu) \\
& =\sum_{m, n} C_{n}^{m} \cos \left(m \lambda-\theta_{n}^{\mathrm{m}}\right) P_{n}^{m}(\mu),
\end{aligned}
$$

where $A_{n}^{m}=C_{n}^{m} \cos \theta_{n}^{m}$ and for $B>0 B_{n}^{m}=C_{n}^{m} \sin \theta_{n}^{m}$. If $\theta_{n}^{m}$ and $C_{n}^{m}$ are assigned at random, then permits the calculation of random vector field. A series of five-day Monte Carlo forecasts were made for a three-month winter period. The forecasts were for the $500 \mathrm{mb}$ height field. The three-month period thus consisted of 18 pentads. The results were given by averaging over these 18 independent calculations. The dynamical component was a spectral barotropic model with triangular truncation at wave number eighteen. A choice of $m=10$ was found to give relatively stable statistical estimates of second moment quantities. The results are shown in figure 6 . Figure 6 gives the RMS error of sample mean and the sample standard deviation for 18 Monte Carlo forecasts. The difference between the respective curves is indicative of the external error growth for this model, as SDP predicts only the internal error growth. As pointed out by Pitcher the model used here was a simple one, however dramatic improvements are not expected even if we use existing operational forecasting models. After a few days the error growth, because of model imperfections, vitiated the advantage obtained by SDP.

It is a common experience of meteorologists that atmospheric flow patterns under certain situations become rather stable from one day to the other

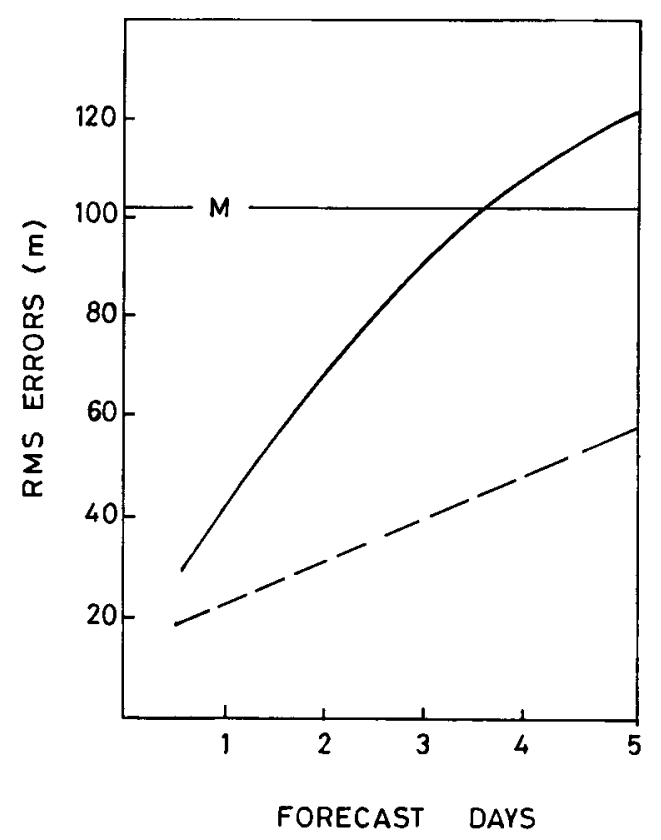

Figure 6. RMS error of sample mean (solid line) and sample standard deviation (dashed line) for 18 Monte Carlo forecasts. The error of a forecast based on climatology is designated by $\mathrm{M}$ (after Pitcher 1979). 
(quasi-persistent) over a limited region. In other words, we can say that the predictability of local synoptic features gets enhanced under special atmospheric conditions. For example, the blocking situation which tends to persist over a region for a few weeks is easily distinguishable from the situations in which short-waves follow each other in quick succession. The former situation becomes a stable flow pattern and is thus a priori more predictable than the less predictable situation characterised by the later situation. Leith (1979) applied the Monte Carlo technique to provide an estimate of the local synoptic scale predictability. He conducted a numerical experiment in which random perturbations for a sample of $m=100$ perturbations, were imposed on the vorticity field and the effect of these perturbations in the forecast through a non-divergent spectral barotropic model was investigated. He found that the error sensitivity on the forecast was higher in the region of maximum wind speed which was expected from the error advection effects.

The Monte Carlo alternative for the solutions of SD equations suggests a promising alternative and further investigation in this direction may lead to its applications to realistic forecasts. Recently another technique called Lagged Average Forecasting (LAF) has been proposed by Hoffman and Kalnay (1983) as an alternative to Monte Carlo forecasting also. The utilization of LAF in stochastic dynamic prediction may make it more feasible for practical forecasting problems.

\section{Conclusions}

The SDP technique is based on some sound principles and has the capability of quantifying the uncertainty in the forecast field caused by the uncertainty in the initial field. However after nearly 15 years since the technique was first suggested, it has been applied only to a single realistic case study and all other studies are based mainly on the applications to the Lorenz's minimum equations. The major factor responsible for this has been the excessive computational time. The enhanced computational time arises because of the simultaneous calculations of large number of covariance parameters. Greater effort is required, even with the simplified situations, to find ways for reducing the computational time so that it may be used for operational purposes.

Depending on the numerical methods used, the simplicity the dynamical models has been put into, the incorrect mathematical representations of certain physical processes, the imperfect parameterisation of sub-grid scale processes, a substantial contribution to the growth in forecast error is unavoidable. This is generally referred as external growth. The SDP technique predicts the internal error growth. The quantitative estimation of uncertainty by SDP may get overmasked by the external error growth also. Hence any attempt in making use of SDP for operational use will have to assess the external growth also.

\section{Acknowledgements}

The authors are thankful to the Director, Indian Institute of Tropical Meteorology for giving his support to the study of SDP techniques reviewed in this paper. Some 
in depth remarks by an anonymous referee made the presentation of the text more clear.

\section{References}

Anthes R A 1982 Tropical Cyclones: their evolution structure and effects (Boston: American Meteorological Society) Chapter 7

Epstein E S 1962 J. Appl. Meteorol. 1169

Epstein E S 1969a Tellus 21739

Epstein E S 1969b J. Appl. Meteorol. 8190

Epstein E S and Pitcher E J 1972 J. Atmos. Sci. 29244

Farago T 1978 Tellus 30335

Fleming R J 1971a Mon. Weather Rev. 99851

Fleming R J 1971b Mon. Weather Rev. 99927

Fleming R J 1973 J. Appl. Meteorol. 12114

Gleeson T A 1968 Proc. Conf. on statistical meteorology (Hartford: Am. Met. Soc.) p 1

Gleeson T A $1970 \mathrm{~J}$. Appl. Meteorol. 2333

Gustafsson N 1981 Dynamic Meteorology, Data Assimilation methods (ed) Bengtsson et al (Berlin: Springer Verlag) p 17

Hoffman R N and Kalnay E 1983 Tellus A35 100

Joshi P C 1981 Proc. Indian Science Congress (Varanasi) Physics Section contribution No. 75

Kruger H B 1969 Q. J. R. Meteorol. Soc. 95.21

Laurman J A 1978 Tellus 30404

Leith C E 1974 Mon. Weather Rev. 102409

Leith C E 1979 Proc. Workshop on stochastic dynamic prediction (UK: European Centre for Medium Range Forecasting) pp 10

Leith C E 1980 Proc. WMO symposium on probabilistic and statistical methods in weather forecasting (Nice, France) pp 33

Lorenz E N 1960 Tellus 12243

Lorenz E N 1963 J. Atmos. Sci. 20130

Lorenz E N 1965 Tellus 17321

Lorenz E N 1976 Proc. of Colloquim on 'Weather forecasting and weather forecasts' I (Colarado: NCAR) pp 214

Lorenz E N 1982 Tellus 34505

Mukherjee T K and Datta R K 1972 Indian J. Meteorol. Geophys. 23345

Orszag S A 1970 J. Fluid Mech. 41363

Otto-Bliesner B, Baumhefner D P and Schlatter T W 1977 Mon. Weather Rev. 1051083

Pitcher E J 1977 J. Atmos. Sci. 343

Pitcher E J 1979 Proc. Workshop on stochastic dynamic prediction (UK: European Centre for Medium Range Forecasting) pp 37

Platzman G W 1964 Tellus 16422

Ramanathan Y, Kulkarni P and Sikka D R 1973 J. Appl. Meteorol. 12977

Sikka D R and Ramanathan Y 1970 Indian J. Meteorol. Geophys. 21238

Sikka D R and Joshi P C 1983 Proc. Indian Society for Theory and Applications of Probability (Calcutta) (in press)

Shukla J and Saha K R 1970 J. Meterol. Soc. (Jpn) 48405

Vincent D G and Borenstein H 1980 Mon. Weather Rev. 1081510

Wiin-Nielsen A 1979 Geophys. Astrophys. Fluid Dynamics 12295 